\title{
Analysis of riluzole's profile of use in a Central Hospital in Lisbon
}

This article was published in the following Dove Press journal:

Patient Preference and Adherence

\author{
Ana Paróla' \\ Fátima da Silva Mousinho \\ de Palhares Falcãol,2 \\ Helena Farinha' \\ André Caetano 3 \\ Luís Santos ${ }^{3}$ \\ Elmira Medeiros ${ }^{3}$ \\ Miguel Viana-Baptista ${ }^{3,4}$ \\ 'Pharmacy Department, Centro \\ Hospitalar de Lisboa Ocidental, \\ Lisbon, Portugal; '2Department of \\ Social Pharmacy, Faculty of Pharmacy, \\ University of Lisbon, Lisbon, Portugal; \\ ${ }^{3}$ Neurology Department, Centro \\ Hospitalar de Lisboa Ocidental, \\ Lisbon, Portugal; ${ }^{4}$ Faculty of Medical \\ Sciences, Nova University of Lisbon, \\ Lisbon, Portugal
}

Purpose: Riluzole is indicated to prolong life or delay the institution of mechanical ventilation in patients with amyotrophic lateral sclerosis (ALS). Clinical studies have shown that this drug prolongs survival, defined as living patients who are not intubated for mechanical ventilation and without tracheotomy. The purpose of this study is to characterize riluzole's use as well as the user population in order to contribute to a rational and safe use.

Patients and methods: Descriptive, observational, retrospective study describing and characterizing the use of riluzole in ALS patients between July 2006 and December 2016 conducted in a Lisbon's Central Hospital.

Results: Over the course of the study period, 77 patients with different phenotypes of ALS received riluzole. The majority of patients $(63 \%, n=49)$ were male. The median survival was 10.1 months, but 12 patients (16\%) remained on therapy for more than 3 years; $65 \%$ of patients were lost to follow-up. The mean adherence rate was $91.2 \%$, and the median adherence rate was $99.3 \%$. One patient discontinued therapy due to gastrointestinal intolerance. Dyspnea and cough were the most common side effects, with roughly one third of patients experiencing each, followed by asthenia and hepatic effects.

Conclusion: Despite the extended enrollment period, only 77 patients met the criteria for study inclusion. Nonetheless, statistical data regarding our population is in accordance with reported international data. High adherence rates were observed, but $14 \%$ of patients discontinued riluzole. In such cases, assessment by a multidisciplinary team is warranted.

Keywords: riluzole, adherence rate, amyotrophic lateral sclerosis, safety profile, drug interactions

\section{Introduction}

Riluzole is included in the group of drugs that act on the nervous system. Although the mechanism of action of riluzole and the pathogenesis of amyotrophic lateral sclerosis (ALS) have not been fully elucidated, it has been suggested that riluzole acts by the inhibition of glutamate-related processes, which are thought to play a role in the cell death that occurs in this disease. Thus, it has been proposed that riluzole pre-synaptically inhibits glutamate release and post-synaptically interferes with the excitatory effect of some amino acids. ${ }^{1-4}$

Clinical studies have shown that this drug prolongs survival - defined as living patients without tracheotomy and without intubation for mechanical ventilation. There is no evidence of riluzole having a therapeutic effect on motor function, pulmonary function, fasciculations, muscle strength, and motor symptoms, or on the final stages of ALS. ${ }^{1,2}$

According to the Summary of the Product's Characteristics (SPCs) approved by the European Medicines Agency (EMA), this drug should not be used in patients with
Pharmacy Department, Hospital de Egas Moniz, Rua Junqueira, 126, 1349-019 Lisboa, Portugal

Tel +35 I 210432608

$\mathrm{Fax}+351210432310$

Email aparola@chlo.min-saude.pt $\mathrm{BY} \mathrm{NC}$ and incorporate the Creative Commons Attribution - Non Commercial (unported, v3.0) License (http://creativecommons.org/licenses/by-nc/3.0/). By accessing the work you
hereby accept the Terms. Non-commercial uses of the work are permitted without any further permission from Dove Medical Press Limited, provided the work is properly attributed. For permission hereby accept the Terms. Non-commercial uses of the work are permitted without any further permission from Dove Mediect
for commercial use of this work, please see paragraphs 4.2 and 5 of our Terms (https://www.dovepress.com/terms.php). 
any other form of motor neuron disease. ${ }^{1,2}$ Nonetheless, the safety and efficacy of riluzole have been studied in other diseases, such as spinocerebellar ataxia or Friedreich's ataxia, with modest results. ${ }^{5}$

Furthermore, the effect of riluzole $50 \mathrm{mg} /$ day was not statistically significant when compared with placebo, and the effect of riluzole $200 \mathrm{mg}$ /day was essentially comparable to that of the $100 \mathrm{mg} /$ day dose of the drug. ${ }^{6,7}$

Riluzole is the only drug licensed in the European Union for ALS. According to the EMA, clinical investigation of medicinal products for treatment of ALS is necessary, especially for disease-modifying treatments. ${ }^{8}$ Unfortunately, massive failures of neuroprotective agents over the past 20 years have been observed, including antiglutamatergic strategies (eg, ceftriaxone), anti-inflammatory strategies (eg, celecoxib), and anti-oxidative strategies (eg, coenzyme Q10), among others. ${ }^{9-11}$

The beneficial effects of edaravone (Radicava $\left.{ }^{\circledR}\right)$, unmarketed in the European Union, have been demonstrated in preclinical trials, as well as in a 6 month, randomized, placebo-controlled, double-blind study conducted in Japanese patients with ALS ( $n=137)$. After the failure of other clinical trials, this trial by Tanaka et al was finally successful in demonstrating the statistical difference on the primary endpoint - the revised ALS Functional Rating Scale score $(P=0.0013) .{ }^{11,12}$ Marketing authorization was initially granted in Japan and, subsequently, in the USA; however, several limitations of this drug were noted, including the fact that the treatment requires continuous 30-minutes long, daily intravenous infusions on a 2-weeks-on, 2-weeks-off schedule. ${ }^{11,12}$

According to the field experts, cell-based therapies may represent a new therapeutic approach for ALS. ${ }^{9}$ Lu Chen et al studied the effectiveness of riluzole in clinical practice and assessed patients every 3 months. In total, 1,540 patients with ALS were recruited between January 2007 and December 2013, of whom 415 (26.9\%) were medicated with riluzole. In this cohort, the use of riluzole was defined as the administration of riluzole for a period of more than 2 weeks. Patients in the riluzole group were divided into three subgroups according to the calculated daily-dose quartiles (dose of riluzole administered to an adult of $70 \mathrm{~kg}$ in 1 day was $100 \mathrm{mg}$ ). No statistically significant difference was found between the control group and the riluzole group with respect to the prognosis of the patients. However, the results seem to suggest that the long-term use of the drug may result in a better prognosis. ${ }^{13}$

There is limited data on therapeutic adherence, lifestyle changes, or non-pharmacological therapy in this population.
However, adherence is a primary determinant of the success of chronic therapy, because low adherence affects the efficacy of the drug. ${ }^{14}$ Therefore, this study was conducted to describe and characterize the use pattern of riluzole in a Central Hospital in Lisbon.

\section{Patients and methods Study design}

This descriptive, observational, retrospective study describing and characterizing the use of riluzole in ALS patients between July 2006 and December 2016 was conducted in Lisbon's Central Hospital.

\section{Population and study sample}

All patients with riluzole-dispensing records for ALS (software program used by the Pharmaceutical Services [CPCGlintt]) were included in the study.

\section{Collection of information}

The collection of demographic, therapeutic, and clinical information was carried out by consulting the hospital records as well as by contacting an attending physician, where justified. The adherence rate was calculated using the frequency of riluzole dispensation, using the formula in Figure 1.

$$
\mathrm{AR}=\frac{\text { Days of dispensed treatment }}{\text { Last dispense }- \text { First dispense }} \times 100
$$

Figure I Adherence rate (AR) calculation.

\section{Statistical analysis}

Data were analyzed using the SPSS V17.0 program, using a $5 \%$ level of significance to indicate statistical significance. The statistical methodology of univariate and bivariate descriptive analyses was applied, where applicable. For the quantitative variables, the measures of central tendency and dispersion were determined. For qualitative variables, the relative and absolute frequencies were determined. The statistical association between the variables was evaluated through $\chi^{2}$ and Fisher's exact tests, as applicable.

\section{Results}

\section{Patient characteristics}

Over the course of the study period, 77 patients with different phenotypes of ALS received riluzole; 75\% had a probable diagnosis of ALS. Diagnostic data was missing only in five patients. Considering an estimated hospital influence area of 950,000 habitants, the calculated prevalence of ALS was $8 / 100,000$. 
The majority of patients $(63 \%, n=49)$ were male. The mean age of the patients was 67 years, but the range spanned from 38 to 89 . Three of these patients (4\%) had young-onset ALS and were 45 years of age or younger. Bulbar onset was noted in 29 patients (38\%), limb-onset in 40 patients $(52 \%)$, and data were missing in eight patients (10\%). Nine (12\%) and thirteen (17\%) patients had a history of drinking and tobacco use, respectively. Patients had a variety of concomitant diseases, with the most common being cardiovascular disease $(65 \%)$ and other nervous system disorders (22\%) (Table 1).

The median survival was 17.9 months $(\mathrm{n}=56)$. In total, 12 patients $(16 \%)$ remained on therapy for more than 3 years, whereas three patients were lost to follow-up. Time delay to diagnosis since the appearance of first symptoms was recorded in the clinical files of 58 patients, and ranged from 1 to 144 months, with an estimated median time delay of 22 months.

Table I Patient comorbidities

\begin{tabular}{ll}
\hline Comorbidity & Number of patients (\%) \\
\hline Cancer & $8(10)$ \\
Endocrine disorder & $20(26)$ \\
Immune disorder & $2(3)$ \\
Blood disorder & $7(9)$ \\
Mental disorder & $14(18)$ \\
Nervous system disorder & $17(22)$ \\
Cardiovascular disease & $50(65)$ \\
Respiratory disorder & $15(19)$ \\
Digestive disorder & $16(21)$ \\
Skin disease & $2(3)$ \\
Muscular disorder & $5(6)$ \\
\hline
\end{tabular}

\section{Riluzole use}

In $75 \%(\mathrm{n}=58)$ of patients, riluzole was first dispensed within 15 days of diagnosis; 76 patients were taking one defined daily dose $(100 \mathrm{mg})$ of riluzole. According to dispensing records, the mean duration of treatment was 18 months, but $60 \%(n=49)$ received less than 12 months of riluzole treatment. Noninvasive ventilation was registered in 33 patients (43\%), of whom 28 remained on riluzole after ventilation. Tracheostomy was registered in four patients $(5 \%)$.

In vitro studies with human liver microsomal preparations suggest that cytochrome CYP1A2 is the major isozyme involved in the oxidative metabolism of riluzole. Thus, CYP1A2 inhibitors (eg, caffeine, diclofenac, diazepam, clomipramine, imipramine, fluvoxamine, theophylline, amitriptyline, and quinolones) may decrease the rate of elimination of riluzole, whereas CYP1A2 inducers (eg, tobacco, rifampicin, and omeprazole) may increase its rate of elimination. ${ }^{1,2,4}$

Of the drugs known to interact with riluzole, we saw omeprazole and amitriptyline most often in this population, in 17 and ten patients, respectively. Diazepam, diclofenac, fluvoxamine, and quinolones each had one patient taking them, whereas clomipramine, imipramine, nicergoline, and rifampicin intake was not seen in these patients.

Dysphagia is a feature of ALS and was seen in 37 patients (Figure 2). In these patients, swallowing riluzole tablets whole could be challenging. Although riluzole can be crushed, it has an anesthetic effect on the tongue when administered thus. ${ }^{15,16}$ Eighteen patients had a percutaneous endoscopic gastrostomy (PEG) tube inserted and, although crushed tablets can be given via the PEG tube, there are safety and efficacy issues that have yet to be elucidated.

The adherence rates of patients were calculated on the basis of the dispensing dates of riluzole, as shown in Figure 1. The mean and median adherence were $91.2 \%$ and $99.3 \%$, respectively. The distribution of adherence rates can be seen in Figure 3. One patient discontinued therapy due to gastrointestinal intolerance, and eleven patients (14\%) discontinued therapy due to poor belief in riluzole's efficacy. Fourteen patients chose to undertake follow-up in other hospitals that were specialized centers for ALS.

In deceased patients, riluzole's last dispensing data were observed approximately 75 days before death.

\section{Suspected adverse reactions}

With regard to riluzole's safety profile, nausea $(12 \%-20 \%)$, abdominal pain $(5 \%-8 \%)$, diarrhea $(5 \%-9 \%)$, alanine aminotransferase (ALT) increases of at least one level above the upper limit of normal (ULN; 50\%) and worsening of asthenia $(14 \%-26 \%)$ were the most commonly reported side effects. ${ }^{3,7}$ Less frequent neurological effects included incoordination,

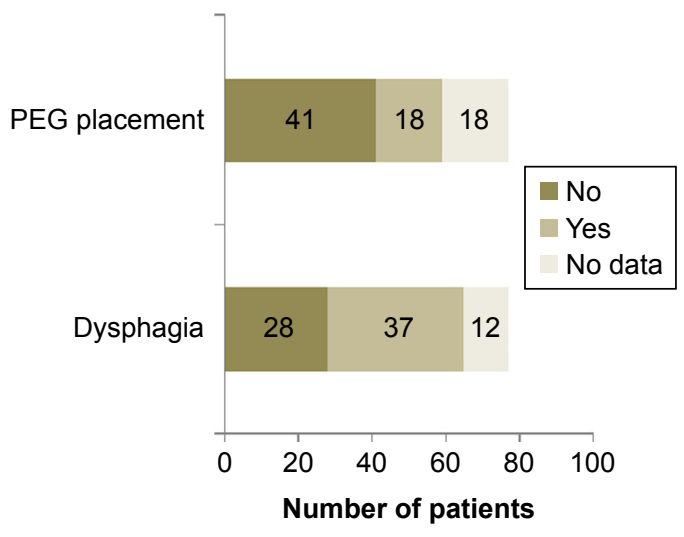

Figure 2 Patients requiring alternative routes of administration. Abbreviation: PEG, percutaneous endoscopic gastrostomy. 


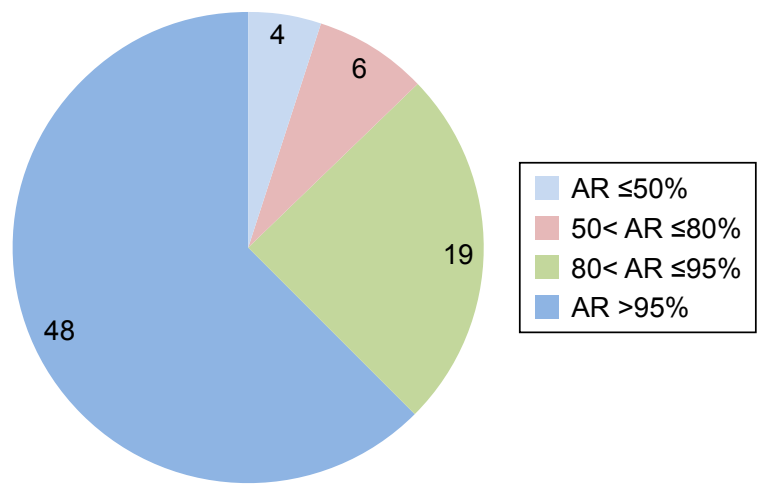

Figure 3 Distribution of adherence rates. Abbreviation: AR, adherence rate.

dizziness $(5 \%-12 \%)$, and depression $(4 \%-6 \%)$, and pulmonary interstitial disease was infrequently observed in patients treated with riluzole. Patients with dry cough and/or dyspnea should, therefore, be evaluated., ${ }^{3,7}$ Serious neutropenia and hepatitis were reported with unknown frequency. A leukogram evaluation is recommended if any febrile illness develops. In clinical trials, the changes in laboratory values occurred most frequently within 2-6 months of initiating therapy. ${ }^{1,2,7}$

Based on the patients' follow-up appointments, a number of suspected adverse drug reactions (ADRs) were noted. Dyspnea and dry cough were the most common, with roughly one third of patients experiencing each, followed by asthenia and hepatic effects (Figure 4).

Clinicians did not attribute some of the patients' complaints to riluzole, and causality was not assessed according to the Naranjo algorithm. Some possible confounding factors may include the data collection method for ADRs. The recommended monitoring includes monthly serum transaminases determination during the first 3 months of treatment, quarterly for the remainder of the first year, and periodically thereafter. ${ }^{1,2}$ Although clinicians perceived that hepatic alterations should be evaluated, we did not find evidence of periodic hepatic function monitoring, in accordance to riluzole's SPC recommendations. Complete missing data were detected in $35 \%$ of the patients. The ALT values ranged from 7 to $105 \mathrm{U} / \mathrm{L}$.

\section{Discussion}

In this retrospective study, an average duration of treatment of 18 months, ranging up to 133 months was verified, as expected from the reported international data. ${ }^{1-3,13,17}$ High adherence rates to riluzole treatment were observed, which suggests that any person who considers himself really ill makes everything possible to survive. However, in the final stages of life, riluzole's last dispensing date was observed approximately 75 days before death, which could be related to physicians' and patients' beliefs regarding riluzole's efficacy.

We observed that $14 \%$ of patients discontinued riluzole. In patients with adherence rates below $80 \%$, assessment by the multidisciplinary team is warranted, and non-adherence factors should be addressed. Disease onset in late adulthood was observed most frequently, and young disease onset accounted for $4 \%$ of the cases. This statistic is in accordance with the international epidemiological data for ALS. ${ }^{17}$

Cognitive and behavioral impairment are now recognized features of ALS. In our population, approximately $20 \%$ of patients had a further nervous system disorder or mental disorder. ${ }^{17}$ Potential drug-drug interactions were identified, but its clinical significance remains unclear. Moreover,

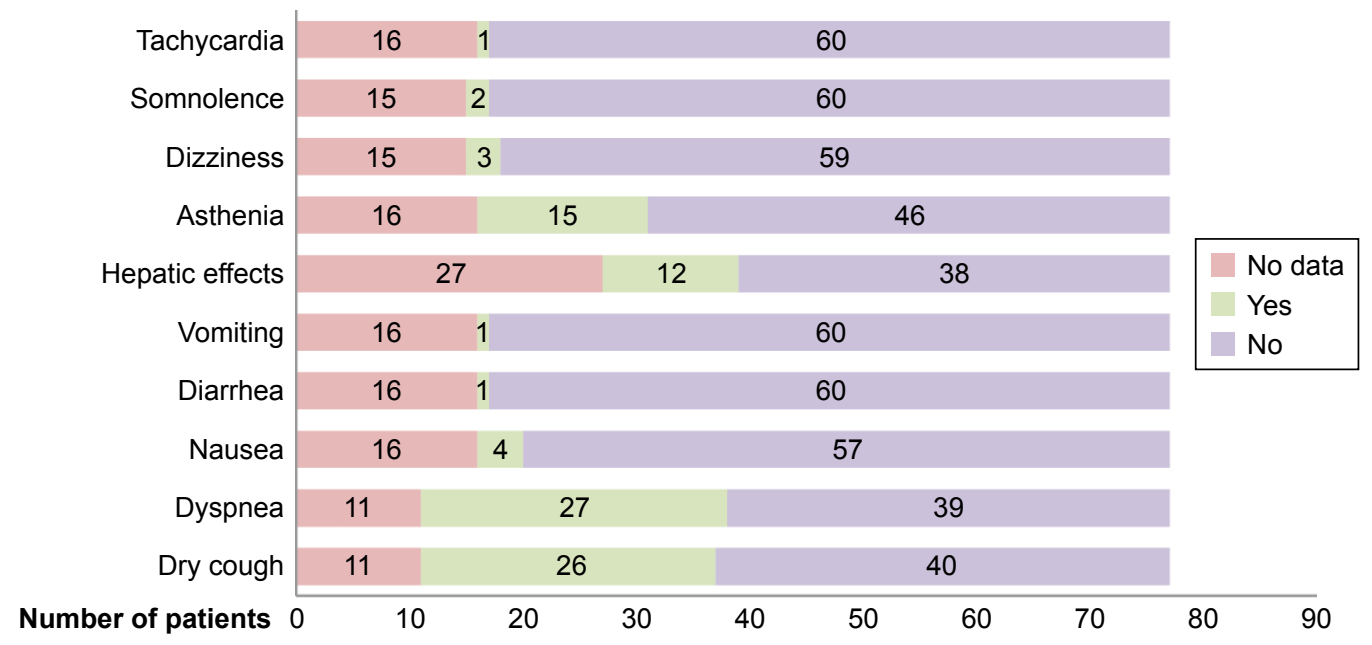

Figure 4 Suspected adverse drug reactions. 
riluzole management in patients with impaired swallowing remains challenging. Although statistical analysis was conducted, we could not recognize any factor (dysphagia, interacting drugs, reported side effects, etc.) that was possibly impacting riluzole's efficacy or safety.

Some limitations of this study include the small sample size and the adherence evaluation and data collection methods. Using dispensing dates to evaluate adherence does not take into consideration other factors that could affect the patient's medication usage, such as a period of hospitalization when the patient was provided with inpatient medication. Lastly, the retrospective nature of the study lends it some inherent disadvantages. Patients were not randomized and are subject to selection bias in that they had to be patients within the Central Hospital in Lisbon. Furthermore, the use of hospital records assumes the accuracy and completeness of these records.

\section{Conclusion}

Despite the extended enrollment period, only 77 patients met the criteria to be included in the study, which indicates the paucity of this disease. Nonetheless, statistical data with regard to our population is in accordance with the reported international data. In addition, the prospect of evaluating data on therapeutic adherence, which is a primary determinant of the success of chronic therapy, and possible ADRs, allowed us to better illustrate the riluzole-use pattern in our hospital. We observed high adherence rates, but $14 \%$ of patients discontinued riluzole due to poor belief in its efficacy. Focus by the multidisciplinary team is to assure optimal adherence rates to the only available, on-label drug for ALS.

Despite the above-stated study limitations, this study allowed the identification of some relevant aspects related with this patient population that the multidisciplinary team can use to plan and develop interventions directed toward patient outcomes, with particular attention to those patients who have associated higher risk factors, which can become a new objective of study and evaluation.

\section{Disclosure}

The authors report no conflicts of interest in this work.

\section{References}

1. Rilutek S.P.Cs. [updated April 14, 2016]. Available from: http:// www.ema.europa.eu/ema/index.jsp?curl=pages/medicines/ human/medicines/000109/human_med_001039.jsp\&mid= WC0b01ac058001d124. Accessed January 11, 2017.

2. Riluzole Zentiva [webpage on the Internet]. London: Europen Medicines Agency; 2012 [updated May 30, 2018]. Available from: http://www.ema.europa.eu/ema/index.jsp?curl=pages/ medicines/human/medicines/002622/human_med_001551. jsp\&mid=WC0b01ac058001d124. Accessed January 11, 2017.

3. Bensimon G, Lacomblez L, Meininger V. A controlled trial of riluzole in amyotrophic lateral sclerosis. ALS/Riluzole Study Group. $N$ Engl J Med. 1994;330(9):585-591.

4. Brites D, Vaz AR. Microglia centered pathogenesis in ALS: insights in cell interconnectivity. Front Cell Neurosci. 2014;8:117.

5. Romano S, Coarelli G, Marcotulli C, et al. Riluzole in patients with hereditary cerebellar ataxia: a randomised, double-blind, placebocontrolled trial. Lancet Neurol. 2015;14(10):985-991.

6. Miller RG, Mitchell JD, Moore DH. Riluzole for amyotrophic lateral sclerosis (ALS)/motor neuron disease (MND). Cochrane Database Syst Rev. 2012;3:CD001447.

7. IBM Micromedex ${ }^{\circledR}$ Web Applications Access [webpage on the Internet]. Armonk, NY: IBM Corporation; 2018. Available from: http://www. micromedexsolutions.com/micromedex $/$ librarian/PFDefaultActionId/ evidencexpert.DoIntegratedSearch\#. Accessed January 11, 2017.

8. Committee for Medicinal Product for Human Use. Guideline on clinical investigation of medicinal products for the treatment of amyotrophic lateral sclerosis. 2015. Available from: http://www.ema.europa. eu/docs/en_GB/document_library/Scientific_guideline/2015/12/ WC500199241.pdf. Accessed April 18, 2018.

9. Mathis S, Couratier P, Julian A, Corcia P, Le Masson G. Current view and perspectives in amyotrophic lateral sclerosis. Neural Regen Res. 2017; 12(2):181-184.

10. de Carvalho M. Do We Have a Channel Solution for ALS? EBioMedicine. 2015;2(12):1842-1843.

11. Petrov D, Mansfield C, Moussy A, Hermine O. ALS Clinical Trials Review: 20 Years of Failure. Are We Any Closer to Registering a New Treatment? Front Aging Neurosci. 2017;9:68.

12. Radicava S.P.Cs. [updated May, 2017]. Available from: https:// www.accessdata.fda.gov/drugsatfda_docs/label/2017/209176lbl.pdf. Accessed April 18, 2018.

13. Chen L, Liu X, Tang L, Zhang N, Fan D. Long-Term Use of Riluzole Could Improve the Prognosis of Sporadic Amyotrophic Lateral Sclerosis Patients: A Real-World Cohort Study in China. Front Aging Neurosci. 2016;8:246.

14. Sabaté E. Adherence to Long-Term Therapies: Evidence for Action. Geneva: World Health Organization; 2003.

15. Onesti E, Schettino I, Gori MC, et al. Dysphagia in Amyotrophic Lateral Sclerosis: Impact on Patient Behavior, Diet Adaptation, and Riluzole Management. Front Neurol. 2017;8(9769):64.

16. Dyer AM, Smith A. Riluzole $5 \mathrm{mg} / \mathrm{mL}$ oral suspension: for optimized drug delivery in amyotrophic lateral sclerosis. Drug Des Devel Ther. 2017; 11:59-64.

17. Turner MR, Hardiman O, Benatar M, et al. Controversies and priorities in amyotrophic lateral sclerosis. Lancet Neurol. 2013;12(3):310-322.
Patient Preference and Adherence

\section{Publish your work in this journal}

Patient Preference and Adherence is an international, peer-reviewed, open access journal that focuses on the growing importance of patient preference and adherence throughout the therapeutic continuum. Patient satisfaction, acceptability, quality of life, compliance, persistence and their role in developing new therapeutic modalities and compounds to optimize

\section{Dovepress}

clinical outcomes for existing disease states are major areas of interest for the journal. This journal has been accepted for indexing on PubMed Central. The manuscript management system is completely online and includes a very quick and fair peer-review system, which is all easy to use. Visit http://www. dovepress.com/testimonials.php to read real quotes from published authors. 